\title{
INFLUÊNCIA DE DIFERENTES COBERTURAS FLORESTAIS NA FAUNA DO SOLO NA FLONA MÁRIO XAVIER, NO MUNICÍPIO DE SEROPÉDICA, RJ
}

\author{
Milton Marques Fernandes ${ }^{1}$, Luis Mauro Sampaio Magalhães ${ }^{2}$, Marcos Gervasio Pereira ${ }^{3}$, \\ Maria Elizabeth Fernandes Correia ${ }^{4}$, Roberto Josino de Brito ${ }^{5}$, Márcia Rodrigues de Moura ${ }^{6}$ \\ ${ }^{1}$ Eng. Florestal, Dr., UFPI, Bom Jesus, PI, Brasil - miltonmf@ gmail.com \\ ${ }^{2}$ Eng. Florestal, Dr., UFRRJ, Seropédica, RJ, Brasil - lmauro@terra.com \\ ${ }^{3}$ Eng. Agrônomo, Dr., UFRRJ, Seropédica, RJ, Brasil - gervasio@ufrrj.br \\ ${ }^{4}$ Bióloga, Dra., Embrapa-Agrobiologia, Seropédica, RJ, Brasil - ecorreia@cnpab.embrapa.br \\ ${ }^{5}$ Eng. Agrônomo, M.Sc, UFRRJ, Seropédica, RJ, Brasil - rjbrito@ bol.com.br \\ ${ }^{6}$ Acadêmica em Engenharia Florestal, UFPI, Bom Jesus, PI, Brasil - marcia86@bol.com.br \\ Recebido para publicação: 29/12/2009 - Aceito para publicação: 04/04/2011
}

\begin{abstract}
Resumo
Este trabalho objetiva avaliar a influência de diferentes coberturas florestais (floresta secundária, plantio de sabiá e plantio de andiroba) sazonalmente na fauna edáfica de um Planossolo Háplico. A fauna do solo foi coletada através de armadilhas do tipo pitt fall durante 7 dias, distribuídas aleatoriamente. Foi monitorada a temperatura do solo com um geotermômetro e a umidade do solo pelo método gravimétrico no dia da instalação das armadilhas. A redução do aporte de serapilheira no outono promove um estresse na condição microclimática na interface solo-serapilheira e de alimento para a fauna edáfica, afetando negativamente a atividade e os índices de Shannon e Pielou. Os plantios de andiroba e sabiá foram similares, e a floresta secundária apresentou pequena similaridade, o que reflete a diversidade florística das áreas. Collembola e Formicidae foram os grupos taxonômicos predominantes nas áreas avaliadas.

Palavras chaves: Ecossistemas florestais; mesofauna do solo; serapilheira.
\end{abstract}

\begin{abstract}
Different forest coverings influence in the soil fauna at Flona Mário Xavier municipality of Seropédica$R J$. This study aims to develop a seasonal evaluation of different forest covering influence (secondary forest, sabiá and andiroba manmade forest) on soil fauna of a Haplic Albaqualf. The soil fauna was collected with pitt fall traps along 7 days and randomly distributed. Temperature and soil wet was observed with a geothermomether and by gravimetric method respectively right in the day of traps setting. Litter intake reduction at autumn promotes a microclimatic stress in soil interface - soil-litter and food for soil fauna, adversely affecting the activity and the Shannon \& Pileau index. Andiroba and Sabiá manmade forest had been similar and the secondary forest presented little similarity, which reflects diversity of the floristic areas. Formicidae and Collembola were the dominant taxonomic groups in the evaluated areas.
\end{abstract}

Keywords: Forest ecosystems; soil mesofauna; litter.

\section{INTRODUÇÃO}

A fauna do solo atua juntamente com fungos e bactérias na decomposição da matéria orgânica, participando diretamente da retenção e reciclagem de nutrientes nos ambientes florestais, sendo a decomposição dessa matéria vegetal morta lenta quando há efeitos negativos sobre a fauna do solo (TEIXEIRA et al., 1998). A abundância e diversidade de comunidades da fauna do solo são indicadores de qualidade e influenciam as propriedades físicas do solo, bem como a taxa de decomposição e ciclagem de nutrientes. O aumento do número de indivíduos de espécies da fauna do solo ocorre pela disponibilidade de melhores condições ambientais, que favorecem a reprodução dos invertebrados, 
conforme relatado por Seeber et al. (2005), e que se revelam por meio do índice de diversidade e do índice de uniformidade (BROWN et al., 2004).

Lopes Assad (1997) cita que a destruição do revestimento vegetal do terreno causa flutuações microclimáticas que expõem os organismos aos excessos de temperatura (exposição ao solo), a ciclo de umedecimento e secagem (estresse hídrico prolongado) e ao fogo (mesmo que atingindo somente a liteira), entre outros fatores.

O conjunto de organismos de um solo é marcado pela sua complexidade, tanto em termos quantitativos quanto em diversidade, podendo ser encontrado em grupos diferenciados por uma vasta amplitude genética e funcional, diretamente relacionados ao tipo de ambiente ao qual se adaptam. Por sua sensibilidade às modificações que ocorrem no ambiente edáfico, o conjunto da fauna do solo pode ser considerado como um indicador das condições encontradas no solo (DORAN; ZEISS, 2000).

Inúmeros são os grupos taxonômicos que compõem a fauna edáfica de invertebrados, e algumas classificações, apoiadas no tamanho e na mobilidade dos organismos, são bem difundidas. Nessa divisão, os animais são classificados em microfauna $(<0,2 \mathrm{~mm})$, mesofauna $(0,2-2 \mathrm{~mm})$ e macrofauna $(>2 \mathrm{~mm})$. A primeira divisão engloba animais ligeiramente mais móveis que a microflora, como nematoides. A mesofauna é constituída por espécies que se movimentam nos poros do solo, nas fissuras e interface entre a liteira e o solo. A macrofauna constitui-se de animais de grande mobilidade, que exercem papel no transporte de materiais no solo (LOPES ASSAD, 1997).

Este trabalho objetivou avaliar a influência de diferentes coberturas florestais (floresta secundária, plantio de sabiá - Mimosa caesalpiniaefolia - e plantio de andiroba - Carapa guianenses) sazonalmente na fauna edáfica de um Planossolo Háplico. Nesse contexto, a hipótese apresentada para o presente estudo é que a fauna edáfica, através de sua composição e estrutura, reflete a diversidade florística e as condições ecológicas do ecossistema florestal.

\section{MATERIAL E MÉTODOS}

O estudo foi realizado no município de Seropédica (RJ), com altitude média de $30 \mathrm{~m}$. O clima da região de estudo é classificado como Aw, de Köppen. A média dos últimos dez anos apresenta uma temperatura média máxima de $32,2{ }^{\circ} \mathrm{C}$, sendo a mínima de $20,3{ }^{\circ} \mathrm{C}$. A temperatura média dos últimos dez anos é de $25,2^{\circ} \mathrm{C}$. O solo da área de estudo é um Planossolo Háplico, conforme descrito em Fernandes (2005).

A avaliação da fauna edáfica foi realizada em três áreas distintas, próximas entre si e nas mesmas condições de solo e relevo, abrangendo as seguintes áreas de cobertura florestal: uma floresta em sucessão secundária espontânea (FS), idade aproximada de 80 anos, com cerca de 60 ha e com ocorrência predominante de 4 espécies (Casearia obliqua, Sparattosperma leucanthum, Eugenia florida $e$ Campomanesia sp.), que, juntas, representaram 70\% do total amostrado (SANTOS, 1999; RODRIGUES, 2006). As outras duas áreas analisadas foram dois plantios realizados em 1946, um de Mimosa caesalpiniaefolia Benth (sabiá) (PM) e outro de Carapa guianenses Aubl. (andiroba) (PA), com espaçamento $2 \times 2 \mathrm{~m}$, em talhão de $1.000 \mathrm{~m}^{2}$ cada.

No ano de 1990, um incêndio queimou cerca de $70 \%$ do plantio de sabiá e $90 \%$ do plantio de andiroba, sendo as árvores mortas, de ambas as espécies, cortadas e deixadas no local. A abertura do dossel possibilitou a invasão de outras espécies, gerando um processo de regeneração secundária (FERNANDES, 2005). Segundo levantamento florístico realizado por Rodrigues (2006), 15 anos após a ocorrência do incêndio foram encontradas 8 espécies no plantio de sabiá, destacando-se as espécies Mimosa caesalpiniaefolia, Erythroxylum pulchrum, Guarea guidonea e Lecythis pisonis, que, juntas, representaram 95,45\% do total levantado. No plantio de andiroba foram encontradas 14 espécies, com predomínio das espécies Piptadenia gonoacantha, Mimosa caesalpiniaefolia, Aureliana fasciculata e Guarea guidonea, representando $82 \%$ dos indivíduos amostrados.

Em cada área foram instaladas e distribuídas aleatoriamente 10 armadilhas do tipo pitt fall, utilizadas para a avaliação da atividade da fauna epígea. Na confecção dos pitt fall, foi utilizado um pote plástico com $11 \mathrm{~cm}$ de diâmetro e 7,5 $\mathrm{cm}$ de profundidade. Também foram utilizados pratos plásticos com $15 \mathrm{~cm}$ de diâmetro, para cobrir os potes plásticos e proteger contra a ação da chuva, fixados no solo com auxílio de palitos de madeira, de forma que não impedissem que a mesofauna fosse capturada. Dentro de cada pote foi colocada uma solução de formol $4 \%$, para preservação da fauna no período de uso do pitt fall. As armadilhas foram colocadas na interface solo-serrapilheira e permaneceram durante sete dias no 
campo. Foi monitorada a temperatura do solo com um geotermômetro e a umidade do solo pelo método gravimétrico no dia da instalação das armadilhas (EMBRAPA), 1997).

Decorrido esse tempo, fez-se a triagem com auxílio de lupa e chaves taxonômicas, com quantificação do número total de indivíduos, cálculo dos índices de diversidade de Shannon e Pielou, riqueza de grupos e atividade (indivíduos/armadilhas/dia).

Foi realizada uma análise multivariada, por meio de componentes principais e técnicas de agrupamento da atividade da fauna edáfica com a temperatura e a umidade do solo. Para testar a similaridade das áreas, foi utilizada uma análise multivariada de agrupamentos entre os índices de atividade e de diversidade da fauna edáfica com as características da serapilheira, do aporte e do solo das áreas estudadas. As coletas da fauna do solo foram realizadas na metade das estações da primavera de 2003, e do verão, outono e inverno de 2004.

\section{RESULTADOS E DISCUSSÃO}

\section{Temperatura e umidade do solo}

Quanto à temperatura, verifica-se que a área de plantio de andiroba apresenta a maior temperatura do solo na primavera, outono e inverno, em comparação às demais áreas (Tabela 1). A área de floresta secundária apresentou a menor temperatura do solo e a maior umidade ao longo do estudo. A umidade do solo aumenta da primavera para o verão e reduz-se com a chegada do inverno nas três áreas estudadas. Já a temperatura do solo decresceu da primavera para o inverno (Tabela 1).

Tabela 1. Temperatura e umidade do solo no período de instalação dos pitt fall nas áreas floresta secundária (FS), plantio de sabiá (PS) e plantio de andiroba (PA).

Table 1. Soil temperature and humidity during the installation of pitt fall in the areas secondary forest (FS), sabiá (PS) and andiroba manmade forest (PA).

\begin{tabular}{ccccccccccc}
\hline & \multicolumn{1}{c}{ Temperatura $\left({ }^{\circ} \mathrm{C}\right)$} & \multicolumn{6}{c}{ Umidade (\%) } \\
\hline & Primavera & Verão & Outono & Inverno & Média & Primavera & Verão & Outono & Inverno & Média \\
\hline FS & 23,5 & 23,0 & 21,7 & 21,0 & 22,3 & 13,2 & 15,0 & 9,2 & 8,9 & 11,6 \\
PS & 23,3 & 23,5 & 23,1 & 20,3 & 22,5 & 7,6 & 9,3 & 6,2 & 5,5 & 7,2 \\
PA & 23,9 & 23,5 & 23,4 & 23,3 & 23,5 & 5,4 & 7,6 & 5,9 & 5,3 & 6,0 \\
\hline
\end{tabular}

\section{Índices de diversidade, uniformidade e riqueza}

De forma geral, a área de plantio de sabiá apresenta o maior valor de diversidade de mesofauna edáfica pelo índice de Shannon, e o plantio de andiroba o menor valor, nas diferentes estações estudadas (Tabela 2). O maior índice de Shannon encontrado nas três áreas foi observado na primavera de 2003, e o menor valor no outono de 2004 (Tabela 2).

Segundo Fernandes (2005), a estação de menor aporte de material decíduo nas áreas durante o período de coleta da mesofauna edáfica foi no outono, que promove uma baixa umidade do solo e oferta de alimento, influenciando uma menor diversidade da mesofauna edáfica pelo índice de Shannon.

Corrêa Neto et al. (2001) encontraram maiores valores de diversidade (Shannon) em uma área de floresta de sucessão secundária espontânea, em comparação a uma área de plantio de eucalipto em um Planossolo Háplico na Floresta Nacional Mário Xavier, Seropédica (RJ), onde a maior adição de material decíduo favoreceu o aumento do número de grupos funcionais e da diversidade da fauna do solo na área de floresta de sucessão secundária espontânea. O mesmo comportamento foi observado neste estudo, em que a floresta secundária apresenta maiores valores de diversidade (Shannon) que as demais áreas.

O plantio de sabiá apresenta o maior valor de índice de Pielou (U) em três das quatro estações avaliadas (Tabela 2). O maior valor de Pielou na primavera nas áreas de floresta secundaria e plantio de andiroba deve-se à maior temperatura do solo, que favorece um aumento uniforme do número de indivíduos por grupo taxonômico sem a dominância de determinado grupo taxonômico, permitindo uma maior uniformidade.

Quanto ao índice de riqueza de grupos na área de floresta secundária nas estações verão, outono e inverno, verificam-se os maiores valores em relação às demais áreas (Tabela 2), o que está relacionado ao maior teor de umidade no solo ao longo do período de estudo (Tabela 1) e à diversidade de espécies 
florestais, que fornecem diferentes fontes de material decíduo. Moço et al. (2005) encontraram maior riqueza da fauna edáfica em duas áreas de floresta em diferentes estágios sucessionais, em comparação a pastagem e plantio de eucalipto, o que se deve à maior diversidade de espécies florestais nas áreas de floresta.

Tabela 2. Índices de diversidade de Shannon $(\mathrm{H})$, Pielou $(\mathrm{U})$, riqueza de grupos $(\mathrm{R})$ e atividade da fauna nas áreas de floresta secundária, plantio de sabiá e plantio de andiroba.

Table 2. Shannon $(\mathrm{H})$, diversity index Pielou (U), wealth group (R) and fauna activity in the areas of secondary forest, sabia and andiroba manmade forest.

\begin{tabular}{lcccc}
\hline Áreas & Primavera & Verão & Outono & Inverno \\
\hline Shannon (H) & & & & \\
\hline Floresta secundária & 0,844 & 0,411 & 0,2995 & 0,6667 \\
Plantio de sabiá & 0,9238 & 0,5021 & 0,2147 & 0,7537 \\
Plantio de andiroba & 0,8103 & 0,3494 & 0,2575 & 0,6204 \\
\hline Pielou (U) & & & & \\
\hline Floresta secundária & 0,728 & 0,3274 & 0,2613 & 0,5985 \\
Plantio de sabiá & 0,7508 & 0,4169 & 0,2061 & 0,6984 \\
Plantio de andiroba & 0,689 & 0,2902 & 0,2852 & 0,5957 \\
\hline Riqueza (R) & & & & \\
\hline Floresta secundária & 4,6439 & 5,2055 & 4,7704 & 5,0065 \\
Plantio de sabiá & 5,7622 & 4,6051 & 3,9582 & 4,6833 \\
Plantio de andiroba & 5,298 & 4,4909 & 2,608 & 4,1252 \\
\hline Atividade (indivíduo/armadilha/dia) & & & & \\
\hline Floresta secundária & 9,00 & 26,32 & 7,58 & 5,64 \\
Plantio de sabiá & 8,54 & 34,47 & 4,80 & 5,21 \\
Plantio de andiroba & 6,27 & 31,25 & 6,90 & 6,62 \\
\hline
\end{tabular}

Observa-se que as áreas de plantio de sabiá e andiroba apresentam comportamento similar nas variações do índice de riqueza durante o período de estudo. Esse comportamento foi observado no índice de Shannon e Pielou, com a redução da primavera para o outono e aumento novamente no inverno.

A maior atividade (indivíduo/armadilha/dia) da fauna do solo foi observada no verão, período de maior umidade do solo (Tabela 2). A menor atividade da fauna edáfica foi observada no outono e inverno (Tabela 2), estações em que ocorre a menor umidade do solo (Tabela 1). O plantio de sabiá apresenta a maior atividade no verão e a menor atividade no outono. A floresta secundária e o plantio de andiroba apresentaram a maior atividade da fauna edáfica no verão e a menor no inverno (Tabela 2).

$\mathrm{O}$ aumento da temperatura e da umidade no verão favorece maior atividade em todas as áreas estudadas, em função do aumento de grupos taxonômicos que apresentam maior mobilidade, como, por exemplo, os Collembolas (Tabelas 1, 2 e 3). Já a redução da temperatura reduz a atividade, devido à migração para as camadas do solo em busca de maior umidade e temperatura ou redução do número de indivíduos de maior mobilidade (Tabelas 1, 2 e 3). De acordo com Dangerfield et al. (1991), Collembola e Formicidae apresentam intensa movimentação na superfície do solo, motivo pelo qual esses dois grupos são os principais responsáveis pelo mesmo padrão observado na atividade da fauna edáfica nas áreas de estudo nas diferentes estações avaliadas. A análise dos componentes principais mostra que a atividade da fauna edáfica está altamente correlacionada com a temperatura e a umidade solo, de acordo com o agrupamento das variáveis, mas demonstra que o comportamento das áreas é diferenciado (Figura 1).

\section{Composição relativa dos grupos taxonômicos}

Os grupos taxonômicos Collembola e Formicidae são os principais grupos taxonômicos observados em todas as estações na floresta secundária. O Collembola teve um predomínio sobre os demais grupos taxonômicos no verão e outono. Oliveira e Franklin (1993) encontraram maior densidade populacional de Collembola em pastagens na Amazônia, como consequência da maior liteira em comparação a pastagens queimadas. 
O Formicidae mostra um predomínio sobre os demais grupos somente no inverno, época em que há maior aporte de material decíduo na área, de acordo com estudo de Fernandes (2005). Na primavera apresenta a composição relativa mais equilibrada dos grupos encontrados, com os grupos taxonômicos Collembola, Díptera, Formicidae e Isopoda apresentando expressiva contribuição (Tabela 3).

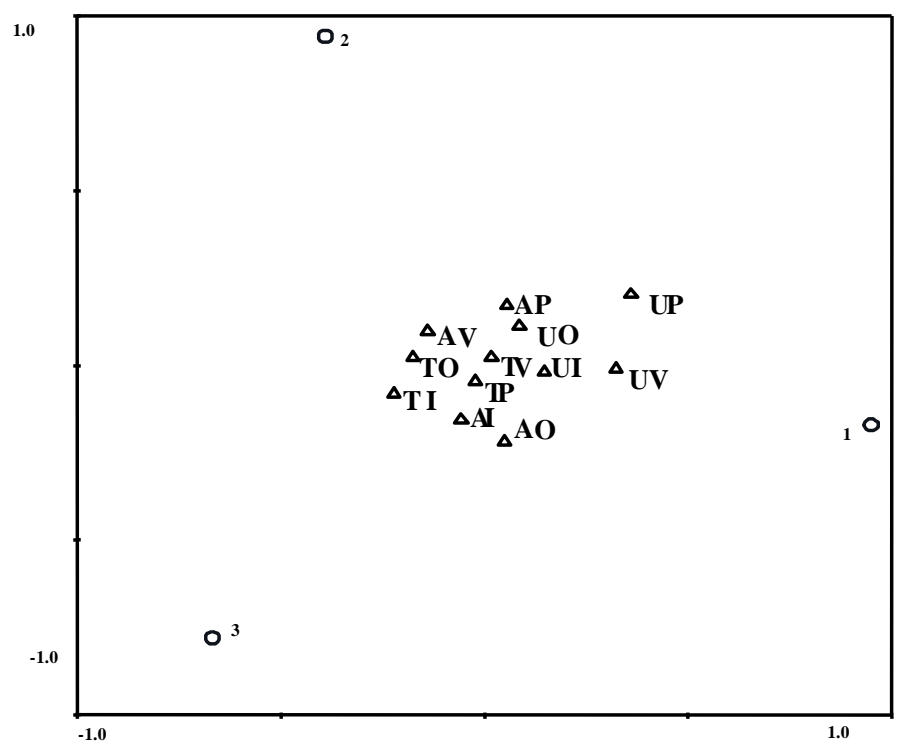

Figura 1. Análise de componentes principais da atividade da fauna edáfica com a temperatura (t), umidade do solo $(\mathrm{U})$ e atividade $(\mathrm{A})$ nas estações climáticas da primavera $(\mathrm{P})$, verão $(\mathrm{V})$, outono $(\mathrm{O})$ e inverno (I), nas áreas de floresta secundária (1), plantio de sabiá (2) e plantio de andiroba (3).

Figure 1. Principal components analysis of the soil fauna activity with temperature (t), soil moisture (U) and activity (A) in the seasons of spring (P), summer (V), autumn (O) and winter (I), in the areas of secondary forest (1), sabia (2) and andiroba manmade forest (3).

Os grupos taxonômicos que apresentam maior percentual no plantio de sabiá são Collembola, Formicidae, Isopoda, Isoptera e Coleóptera (Tabela 3), sendo que Collembola é o grupo de maior percentual no verão e outono. O grupo Formicidae é dominante no inverno, de acordo com Fernandes (2005). É a época de maior deposição de material decíduo, tendo um comportamento semelhante ao da floresta secundária. Já na primavera não ocorre um predomínio de um grupo taxonômico sobre os outros, com Collembola, Isopoda, Coleóptera e Isoptera sendo os grupos com maior percentual. A fauna edáfica da área de andiroba exibe comportamento similar ao das áreas de floresta secundária e sabiá, com predomínio de Collembola no verão e outono e de Formicidae no inverno e uma composição relativa dos grupos taxonômicos na primavera (Tabela 3).

As ordens Collembola e Formicidae foram, dentre todos os grupos taxonômicos, os de maior abundância, correspondendo a cerca de $70 \%$ da comunidade da fauna do solo encontrada nas três áreas estudadas, demonstrando a forte dominância desses dois grupos taxonômicos. Porém há de se destacar que o grupo taxonômico Collembola correspondeu nas três áreas a pelo menos $50 \%$ do total dos indivíduos quantificados (Tabela 3). Huber (2003) ressalta que o hábito alimentar dos Collembola se dá na superfície dos resíduos orgânicos e comumente o número desses organismos é bem mais expressivo na serapilheira que no solo. A dominância do grupo taxonômico Collembola demonstra a importância desses organismos na ciclagem de nutrientes na decomposição da serapilheira na interface solo-serapilheira nas três áreas.

\section{Similaridade}

A análise de agrupamento foi realizada com a fauna edáfica de cada área em conjunto com os parâmetros das características da serapilheira, do aporte do material decíduo e do solo, extraídos de 
Fernandes (2005). A análise de agrupamento evidenciou similaridade entre as áreas de plantios de andiroba e de sabiá, formando um agrupamento separado da floresta secundária (Figura 2). Embora a área de floresta secundária não apresente similaridade pela análise de agrupamento, a distância de ligação é pequena (aproximadamente 12\%). As áreas de plantio de andiroba e o plantio de sabiá apresentam uma distância de ligação pequena de apenas 3\%, evidenciando que as áreas são muito similares.

Tabela 3. Composição relativa dos grupos taxonômicos em percentual (\%) de maior ocorrência na área de floresta secundária e nos plantios de sabiá e andiroba em todas as estações estudadas.

Table 3. Membership on the taxonomic groups of higher occurrence in the area of secondary forest and sabia and andiroba manmade forest in all studied seasons.

\begin{tabular}{lcccc}
\hline Grupos taxonômicos & Primavera-2003 & Verão-2004 & Outono-2004 & Inverno-2004 \\
\hline Floresta secundária & & & & \\
\hline Collembola & 29,37 & 68,86 & 58,19 & 19,75 \\
Formicidae & 23,49 & 24,20 & 29,38 & 48,35 \\
Díptera & 14,76 & 1,14 & 3,20 & 5,06 \\
Isopoda & 11,43 & 1,03 & 0,39 & 0 \\
Outros & 20,95 & 4,77 & 8,84 & 26,84 \\
\hline Sabiá & & & & \\
\hline Collembola & 22,24 & 69,14 & 68,15 & 8,77 \\
Formicidae & 9,03 & 15,04 & 22,32 & 39,18 \\
Díptera & 9,03 & 2,93 & 2,08 & 5,48 \\
Isopoda & 14,55 & 4,70 & 0,89 & 4,93 \\
Coleoptera & 21,24 & 0,61 & 1,49 & 18,08 \\
Outros & 23,91 & 7,58 & 5,07 & 23,56 \\
\hline Andiroba & & & & \\
\hline Collembola & 23,23 & 79,75 & 59,83 & 28,88 \\
Formicidae & 28,47 & 12,48 & 24,84 & 42,67 \\
Díptera & 5,92 & 1,97 & 2,28 & 0,86 \\
Isopoda & 3,64 & 1,05 & 1,86 & 7,11 \\
Outros & 20,66 & 4,75 & 11,19 & 20,48 \\
\hline
\end{tabular}

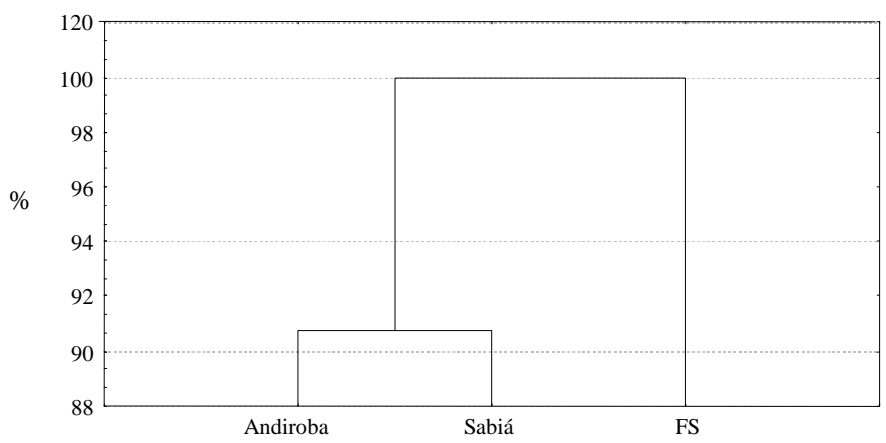

Figura 2. Dendrograma apresentando a distância entre as áreas a partir da análise das comunidades da fauna e as características da serapilheira e do solo com base em distância euclidiana.

Figure 2. Dendrogram showing the distance between the areas by means fauna communities analisys and the litter and soil characteristics, based on euclidean distance.

Rodrigues (2006) avaliou a composição florística das mesmas áreas deste trabalho e observou que as áreas de plantio de sabiá e de andiroba apresentam maior similaridade entre si e que a floresta secundária apresenta uma pequena similaridade. Dessa forma, a similaridade da florística das áreas está de acordo com as características da fauna edáfica, da serapilheira, do aporte de material decíduo e do solo (Figura 2). 


\section{CONCLUSÕES}

- A redução do aporte de serapilheira no outono, conforme estudo realizado nessas áreas, promove um estresse nas condições microclimáticas na interface solo-serapilheira e de alimento para a fauna edáfica, afetando negativamente na atividade e índices de Shannon e Pielou.

- O plantio de andiroba e o plantio de sabiá apresentaram uma grande similaridade, e a floresta secundária pequena similaridade, o que reflete a similaridade florística das áreas.

- Collembola e Formicidae foram os grupos taxonômicos predominantes nas áreas avaliadas, destacando-se que os Collembolas têm um papel importante na decomposição na interface soloserapilheira, e os Formicidae são extremamente móveis, podendo se deslocar para camadas mais superficiais na época de maior deposição de serapilheira.

\section{REFERÊNCIAS}

BROWN, G. G.; MORENO, A. G.; BAROIS, I.; FRAGOSO, C.; ROJAS, P.; HERNÁNDEZ, B.; PATRÓN, J. C. Soil macrofauna in SE Mexican pastures and the effect of conversion from native to introduced pastures. Agriculture, Ecosystems and Environment, v. 103, p. 313 - 327, 2004.

CORRÊA NeTO, T. A.; PEREIRA, M. G.; CORREA, M. E. F.; ANJOS, L. H. C. Deposição de serapilheira e mesofauna edáfica em áreas de eucalipto e floresta sucessão secundária espontânea. Revista Floresta e Ambiente, v. 8, n. 1, p. 70 - 75, 2001.

DANGERFIELD, J. M. Abundance, biomass and diversity of soil macrofauna in Savanna woodland and associated managed habitats. Pedobiologia, p. 141, 1991.

DORAN, J. W.; ZEISS, M. R. Soil health and sustainability: managing the biotic component of soil quality. Applied Soil Ecology, v. 15, p. 3 - 11, 2000.

EMPRESA BRASILEIRA DE PESQUISA AGROPECUÁRIA (EMBRAPA). Manual de métodos de análise de solo. Rio de Janeiro: EMBRAPA/CNPS, 1997. 212 p.

FERNANDES, M. M. Influência da cobertura vegetal nos atributos e na ciclagem de nutrientes em áreas da Floresta Nacional Mário Xavier, Seropédica, RJ. 85 f. Dissertação (Programa de PósGraduação em ciências Ambientais e Florestais) - Universidade Federal Rural do Rio de Janeiro, Seropédica, 2005.

HUBER, A. C. K. Estudos da mesofauna (ácaros e colêmbolos) e macrofauna (minhocas) no processo da vermicompostagem. 90 f. Dissertação (Mestrado em Agronomia - Solos) - Universidade Federal de Pelotas, Pelotas, 2003.

LOPES ASSAD, M. L. Fauna do solo. In: VARGAS, M. A. T.; HUNGRIA, M. (Ed.) Biologia dos solos do cerrado. Planaltina: EMBRAPA, CPAC, 1997. cap. 7, p. 363 - 444.

MOÇO, M. K. S.; GAMA-RODRIGUES, E. F.; GAMA-RODRIGUES, A. C.; CORREIA, M. E. F. Caracterização da fauna edáfica em diferentes coberturas vegetais na Região Norte Fluminense. Revista Brasileira de Ciência do Solo, v. 29, p. 555 - 564, 2005.

OLIVEIRA, E. P.; FRANKLIN, E. Efeito do fogo sobre a mesofauna do solo: recomendações em áreas queimadas. Pesquisa Agropecuária Brasileira, Brasília, v. 28, n. 3, p. 357 - 369, 1993.

RODRIGUES, R. M. M. Regeneração e estrutura de áreas naturais e revegetadas, na Floresta Nacional Mário Xavier, Seropédica, RJ. 70 f. Dissertação (Mestrado em Ciências Ambientais e Florestais) - Universidade Federal Rural do Rio de Janeiro, Seropédica, 2006.

SANTOS, L. A. F. Floresta Nacional Mário Xavier: uma proposta de planejamento ambiental. 299 f. Dissertação (Mestrado em Ciências Ambientais e Florestais) - Universidade Federal Rural do Rio de Janeiro, Seropédica, 1999. 
SEEBER, J.; SEEBER, G. U. H.; KÖSSLER, W.; LANGEL, R.; SCHEU, S.; MEYER, E. Abundance and trophic structure of macrodecomposers on alpine pastureland (Central Alps, Tyrol): effects of abandonment of pasturing. Pedobiologia, v. 49, p. 221 - 228, 2005.

TEIXEIRA, L. B.; SILVA, A. B.; LEITÃO, P. S. Diversidade de invertebrados no solo com diferentes coberturas vegetais no Nordeste Paraense. Belém: EMBRAPA, CPATU, 1998. 22 p. 